\title{
Analyzing and validating the prognostic value and mechanism of colon cancer immune microenvironment
}

\author{
Xinyi Wang ${ }^{1,2 \dagger}$, Jinzhong Duanmu ${ }^{1 \dagger}$, Xiaorui Fu ${ }^{1,2}$, Taiyuan $\mathrm{Li}^{1}$ and Qunguang Jiang ${ }^{1 *}$ (D)
}

\begin{abstract}
Background: Colon cancer is a disease with high malignancy and incidence in the world. Tumor immune microenvironment (TIM) and tumor mutational burden (TMB) have been proved to play crucial roles in predicting clinical outcomes and therapeutic efficacy, but the correlation between them and the underlying mechanism were not completely understood in colon cancer.
\end{abstract}

Methods: In this study, we used Single-Sample Gene Set Enrichment Analysis (ssGSEA) and unsupervised consensus clustering analysis to divide patients from the TCGA cohort into three immune subgroups. Then we validated their differences in immune cell infiltration, overall survival outcomes, clinical phenotypes and expression levels of HLA and checkpoint genes by Mann-Whitney tests. We performed weighted correlation network analysis (WGCNA) to obtain immunity-related module and hub genes. Then we explored the underlying mechanism of hub genes by gene set enrichment analysis (GSEA) and gene set evaluation analysis (GSVA). Finally, we gave an overall view of gene variants and verified the correlation between TIM and TMB by comparing microsatellite instability (MSI) and gene mutations among three immune subgroups.

Results: The colon cancer patients were clustered into low immunity, median immunity and high immunity groups. The median immunity group had a favorable survival probability compared with that of the low and high immunity groups. Three groups had significant differences in immune cell infiltration, tumor stage, living state and T classification. We got 8 hub genes (CCDC69, CLMP, FAM1 10B, FAM129A, GUCY1B3, PALLD, PLEKHO1 and STY11) and predicted that immunity may correlated with inflammatory response, KRAS signaling pathway and T cell infiltration. With higher immunity, the TMB was higher. The most frequent mutations in low and median immunity groups were APC, TP53 and KRAS, while TTN and MUC16 showed higher mutational frequency in high immunity group.

Conclusions: We performed a comprehensive evaluation of the immune microenvironment landscape of colon cancer and demonstrated the positive correlation between immunity and TMB. The hub genes and frequently mutated genes were strongly related to immunity and may give suggestion for immunotherapy in the future.

Keywords: Colon cancer, Tumor immune microenvironment, Tumor mutational burden, Weighted correlation network analysis

\footnotetext{
*Correspondence: fbron.student@sina.com

${ }^{+}$Xinyi Wang and Jinzhong Duanmu contributed equally to this work 1 Department of Gastrointestinal Surgery, The First Affiliated Hospital of Nanchang University, Nanchang, Jiangxi, People's Republic of China Full list of author information is available at the end of the article
}

\section{Background}

Colon cancer is one of the most malignant tumors worldwide [1]. Thanks to the progression in systemically medical treatment and surgical techniques, prognosis of patients with colon cancer has dramatically improved if they are diagnosed at early stage [2]. Prognostic

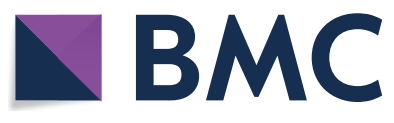

(c) The Author(s) 2020. This article is licensed under a Creative Commons Attribution 4.0 International License, which permits use, sharing, adaptation, distribution and reproduction in any medium or format, as long as you give appropriate credit to the original author(s) and the source, provide a link to the Creative Commons licence, and indicate if changes were made. The images or other third party material in this article are included in the article's Creative Commons licence, unless indicated otherwise in a credit line to the material. If material is not included in the article's Creative Commons licence and your intended use is not permitted by statutory regulation or exceeds the permitted use, you will need to obtain permission directly from the copyright holder. To view a copy of this licence, visit http://creativeco mmons.org/licenses/by/4.0/. The Creative Commons Public Domain Dedication waiver (http://creativecommons.org/publicdomain/ zero/1.0/) applies to the data made available in this article, unless otherwise stated in a credit line to the data. 
prediction of patients with colon cancer mainly relies on the TNM staging system, histopathological criteria, molecular markers and tumor-cell differentiation [3]. Nowadays, accumulated studies have demonstrated the role of gene mutation status, gene expression levels and signaling pathway changes in tumor progression and malignization, but it is still a challenge to find out prognostic factors which can also provide targets for therapy $[4,5]$. The viewpoint that the immune system can influence the progression of cancer has been the hotspot for study over a century. Recently, numerous evidences indicate that the tumor immune microenvironment (TIM) is of great value in predicting prognosis and evaluating therapeutic efficacy factors [6]. TIM is composed of immune cells, immune-related pathways and cytokines that secreted by immune cells. In colon cancer, there has been studies showed that adaptive immune reaction is strongly correlated with survival outcomes and recurrence, and the infiltration of different kinds of immune cells might construct a favorable or unfavorable environment for tumor cells to proliferate and metastasize [7].

Since immune checkpoint-inhibiting agents, such as programmed death-1 receptor (PD1) and cytotoxic T-lymphocyte antigen 4 (CTLA-4) inhibitors, have been developed as antitumor drugs, immunotherapy has become a promising field of cancer treatment and demonstrated its impressive clinical value in patients across multiple types of solid tumors $[8,9]$. Lymphocyte activation gene-3 (LAG3) is another immunotherapy target in the clinic, whose up-regulation is required to prevent the onset of autoimmunity. Sustained antigen exposure in the TIM leads to up-regulated LAG3 expression, resulting in exhaustion of immune cell proliferation and cytokine production [10]. Recently, many studies proved that the expression levels of Indoleamine 2, 3-dioxygenase 1 (IDO1) plays an important role in engender immune tolerance and pathogenic inflammatory processes, which highlights its strong association with T-cell infiltration [11]. The essence of tumor immunotherapy is to arouse and strengthen the immune system to kill tumor cells in various ways. Tumor mutational burden (TMB) was defined as the total amount of coding errors of somatic genes, base substitutions, insertions or deletions detected across per million bases [12]. If TMB is larger, the cancer cell is more mutated, and it is easier for immune cells to recognize and kill it [13]. And tumors which respond to immune checkpoint-inhibiting agents have a higher level of immune cell infiltration and exhibit a T-cell inflamed phenotype. There is a certain correlation between TIM and TMB, and exploring this correlation is of great significance for us to select immunotherapeutic drugs and explore new immunotherapeutic targets [14]. KRAS and BRAF mutational status have been considered as prognostic factors in colon cancers with MSI and may give clues for adjuvant therapy in the future [15-17]. Lin et al. have reported that activation of STAT3 plays a significant role in increasing infiltration with $\mathrm{CD}^{+}$lymphocytes and inhibiting the recruitment of T-regs that enhance colon tumor progression and immune escape [18]. There were several researches which explored the characteristics of TIM in pan-cancer and evaluate the correlation between the landscape of TIM and prognosis of patients, but they were focused on comparing different cancer types in immune cell infiltrating. As a result, further exploration of TIM in genetic level is of great significance.

In this study, by applying unsupervised consensus clustering analysis, we divided patients from the TCGA cohort into three groups (high, median and low immunity) according to ssGSEA scores. Furthermore, we validated their differences in immune cell infiltration, overall survival outcomes, clinical phenotypes and expression levels of HLA and checkpoint genes. In order to screen out essential genes for constructing colon cancer immune microenvironment, we performed WGCNA and got 8 hub genes which were in the module correlated with immune capacity. Finally, we explored the underlying mechanism of hub genes by GSEA and GSVA, and verified the correlation between TIM and TMB to give ideas for immunotherapy of patients with colon cancer.

\section{Methods \\ Data download}

The transcriptome data, somatic mutation data and clinical information of colon cancer patients were obtained from the TCGA database via the GDC data portal (https ://portal.gdc.cancer.gov/repository). We downloaded RNA-seq (level 3, HTSeq-FPKM data) of 445 colon cancer patients [445primarytumortissueand41solidnormaltissue] with complete clinical information from the TCGA database. The clinical information of patients from TCGA database are summarized in Table 1. We downloaded "Masked Somatic Mutation" subtype of somatic mutation data and used the VarScan software to process it. We used a R package called "maftools" [19] to analyze and visualize the Mutation Annotation Format (MAF) of somatic variants. Human Protein Atlas (https://www. proteinatlas.org) was used to validate expression levels of hub genes by immunohistochemistry. MSI information (MSI-H, MSI-L or MSS) for each TCGA samples were obtained from a previous study by Liu et al.[20]. The annotations of genes were obtained from Uniprot database (https://www.uniprot.org/). 
Table 1 Clinical characteristics of the included TCGA dataset

\begin{tabular}{|c|c|c|}
\hline \multicolumn{2}{|l|}{ Characteristics } & \multirow{2}{*}{$\begin{array}{l}\text { Total TCGA } \\
\mathbf{N}\end{array}$} \\
\hline Age years & $<60$ & \\
\hline & $\geq 60$ & 312 \\
\hline \multirow[t]{2}{*}{ Gender } & Male & 212 \\
\hline & Female & 233 \\
\hline \multirow[t]{5}{*}{$\mathrm{T}$} & $\mathrm{T} 1$ & 10 \\
\hline & $\mathrm{T} 2$ & 76 \\
\hline & T3 & 302 \\
\hline & T4 & 56 \\
\hline & Unknown & 1 \\
\hline \multirow[t]{3}{*}{ M } & MO & 328 \\
\hline & M1 & 61 \\
\hline & Unknown & 56 \\
\hline \multirow[t]{4}{*}{ N } & No & 264 \\
\hline & N1 & 102 \\
\hline & N2 & 79 \\
\hline & Unknown & 0 \\
\hline \multirow[t]{5}{*}{ Stage } & Stage I & 75 \\
\hline & Stage II & 174 \\
\hline & Stage III & 124 \\
\hline & Stage IV & 61 \\
\hline & Unknown & 11 \\
\hline \multirow[t]{3}{*}{ Lymphatic invasion } & No & 245 \\
\hline & Yes & 159 \\
\hline & Unknown & 41 \\
\hline \multirow[t]{3}{*}{ Venous invasion } & No & 292 \\
\hline & Yes & 95 \\
\hline & unknown & 58 \\
\hline \multirow[t]{2}{*}{ Fustat } & Alive & 351 \\
\hline & Dead & 94 \\
\hline
\end{tabular}

Implementation of Single-Sample Gene Set Enrichment Analysis ssGSEA

We obtained the marker gene sets for immune cells and immune pathways from another article [21]. We performed ssGSEA to derive the enrichment score of each immune-related term using a R package called "GSVA" [22]. The ssGSEA applies gene signatures expressed by immune cell populations and immune pathways to every cancer samples. The computational approach used in our study included immune cells types and immune pathways that are involved in innate immunity and adaptive immunity. We obtained 29 immune gene sets from several literatures, including immune cell types and functions [23], tumor-infiltrating lymphocytes (TILs) [24], proinflammatory [25], para-inflammation (PI) [26], cytokine and cytokine receptor (CCR)
[27], human leukocyte antigen (HLA) [28], regulatory T (Treg) cells [29], immune checkpoint [30].

\section{Identification of immune subgroups by consensus clustering}

To investigate the correlation between immunity and clinical phenotypes in colon cancer, we clustered colon cancer samples from TCGA into 3 different groups (high, medium and low immunity) with "Consensus Cluster Plus" (50 iterations, resample rate of $80 \%$ ) based on enrichment scores of immune terms in ssGSEA. In order to validated that those 3 subgroups are different in immunity, we use a R package called "estimate" to calculate the immune score, stromal score and ESTIMATE score of every tumor sample [31]. And we compared tumor purity of samples in 3 subgroups by Mann-Whitney U test.

\section{Analysis of clinical information and immunotherapy-related genes}

The Chi-square test was performed to analyze the correlation between immunity and clinical phenotypes, including gender, age, venous invasion, lymphatic invasion, stage, TNM classification and survival state. We classified the total TCGA cohort into subgroups based on clinical phenotypes: gender (male/female), age $(>60 / \leq 60)$, venous invasion (yes/no), lymphatic invasion (yes/no), stage (stage $1+$ stage $2 /$ stage $3+$ stage 4$), \quad \mathrm{T}(\mathrm{T} 1+\mathrm{T} 2 /$ $\mathrm{T} 3+\mathrm{T} 4), \mathrm{N}(\mathrm{N} 0 / \mathrm{N} 1+\mathrm{N} 2), \mathrm{M}(\mathrm{M} 0 / \mathrm{M} 1)$. And we analyzed the difference in overall survival rate between 3 immune subgroups in clinical subgroups by a $R$ package called "survival". The expression level of human leukocyte antigens (HLA) and checkpoint-related genes in 3 immune subgroups were compared by Mann-Whitney test. The proportions of the 22 tumor infiltrating immune cells in 3 immune subgroups were determined by Kruskal-Wallis tests using a R package called "CIBERSORT” [32].

\section{Construction of co-expression module networks}

The Weighted correlation network analysis (WGCNA) was used to construct the gene co-expression network to find clinical-phenotype-related modules and hub genes by the R package "WGCNA"[33]. All genes and samples were filtered by good genes or good samples test. Filtered genes were used to construct a scale-free network by calculating the connection strength between genes. Scale-free $\mathrm{R}^{2}$ ranging from 0 to 1 was used to determine a scale-free topology model. To minimize effects of noise and spurious associations, the adjacency matrix was transformed into Topological Overlap Matrix (TOM). And TOM-based dissimilarity was used to form modules by dynamic tree cut. Here, we set minimal module size as 30 and cut height as 0.25 . We evaluated the correlation 
among module eigengenes (MEs), clinical traits and modules which are related to the traits. For each module, gene significance (GS) and module membership (MM) were calculated and used for hub gene selection. Moreover, Kyoto Encyclopedia of Genes and Gene Ontology (KEGG) pathway enrichment analyses and Gene Ontology (GO) analysis were performed for genes in the modules using the KOBAS database. The cutoff criteria set as $\mathrm{p}$ value $<0.05$.

\section{Predicting underlying mechanism of immunity-related modules and hub genes}

Wilcox test was performed to compare the expression level of hub genes in normal samples colon cancer samples with different clinical phenotypes. We performed GSEA [22] and GSVA to explore correlated pathways of our immune-related risk signature. Gene ontology gene sets "h.all.v7.0.symbols.gmt" were downloaded from Molecular Signatures Database (MSigDB, https://softw are.broadinstitute.org/gsea/downloads.jsp) and were used for the enrichment analysis. When the false discovery rate (FDR) was less than 0.25 , the enriched gene set was considered to be statistically significant. We demonstrated the correlation between hub genes expression levels and immune cells infiltration by calculating the Person correlation coefficients, which was performed by using TIMER database (https://cistrome.shinyapps. io/timer). The function of hub-genes was analyzed by Metascape database (http://metascape.org/) [34].

\section{Calculation of TMB scores and prognostic analysis}

In our study, we calculated the mutation frequency with number of variants/the length of exons for each sample via Perl scripts based on the JAVA8 platform. We classify the colon cancer samples into low-TMB and high-TMB groups according the median data. Mann-Whitney test was conducted to compare the TMB difference among 3 immune subgroups. The survival curves for the prognostic analysis were generated via the Kaplan-Meier method and log-rank tests were utilized to identify significance of differences.

\section{Results}

\section{Immune microenvironment landscape of colon cancer}

The immunity of tumor samples was assessed by applying the ssGSEA approach to the transcriptomes of TCGA colon cancer samples. 29 immune-related pathways and infiltrating immune cells were incorporated to estimate the immune capacity of colon cancer tissues (Fig. 1a). The total TCGA cohort were clustered into 3 subgroups (low immunity: 136 samples, median immunity: 206 samples, high immunity: 103 samples) by applying unsupervised consensus clustering analysis (Fig. 1b-d). To validate the immunity of 3 immune subgroups, we also showed the ESTIMATE score, immune score and stromal score in the heatmap. The association of immunity and colon cancer patients' prognosis was indicated by comparing survival rates of 3 immune subgroups in different clinical subgroups (Fig. 1e-k, Additional file 1: Figure S1). The result showed that survival rates of 3 immune groups have statistical difference in the total TCGA cohort $(\mathrm{P}=0.004)$, age $<60 \quad(\mathrm{P}=0.019)$, no lymphatic invasion $(\mathrm{P}=0.041)$, M0 $(\mathrm{P}=0.024)$, N0 $(\mathrm{P}=0.018)$, stage $1+$ stage $2(\mathrm{P}=0.026)$ and $\mathrm{T} 3+\mathrm{T} 4(\mathrm{P}=0.014)$. In all of these clinical subgroups, patients with median immunity have the best prognosis while patients with lowest immunity have the worst prognosis. The Chi-square test (Additional file 2: Figure S2) showed that immunity classification was correlated with stage $(\mathrm{P}<0.001)$, metastasis $(\mathrm{P}<0.001), \mathrm{N}$ classification $(\mathrm{P}<0.01)$ and survival state $(\mathrm{P}<0.05)$. This demonstrated that immunity could have strong correlation with clinical phenotypes and also serve as a prognostic factor in colon cancer.

\section{immune subgroups are different in immune cell infiltration and expression of immunotherapy-related genes}

To explore the biological behaviors among these immune subtypes, we performed GSVA enrichment analysis. As shown in Fig. 2a, b, Immunity-L was related to immune suppression biological process. Immunity- $\mathrm{M}$ was enriched in stromal and carcinogenic activation pathways such as TGF beta signaling pathway, apoptosis, VEGF and MAPK signaling pathways. Immunity- $\mathrm{H}$ was associated with immune activation including the activation of chemokine signaling pathway, cytokine-cytokine receptor interaction, $\mathrm{T}$ cell receptor signaling pathway and Natural killer cell mediated cytotoxicity. These 3 immune subgroups were also significantly different in tumor purity: the high immunity group has the lowest tumor purity and the low immunity group has the highest tumor purity (Fig. 2c). The fraction of 20 types of infiltrating immune cells were compare among 3 immune subgroups (Fig. 2d). The result showed that 11 types of immune cells, including B cell, macrophages M1, macrophages M2, macrophages M3, resting mast cell, activated mast cell, NK cell, plasma cell, CD4 T cell, CD8 T cell and T cell follicular helper, had significantly different infiltrating levels in different immune subgroup. The expression level of 19 HLA genes were all significantly different among 3 immune subgroups (Fig. 2e). With higher immunity, the expression level of HLA genes was higher. We chose 4 immune checkpoint genes which are regarded as targets in immunotherapy, including CTLA4, IDO1, LAG3 and PDCD1(PD-1). And we found that the expression level of all these genes are highest in high immunity group and lowest in low immunity group (Fig. 2f-i). Median 


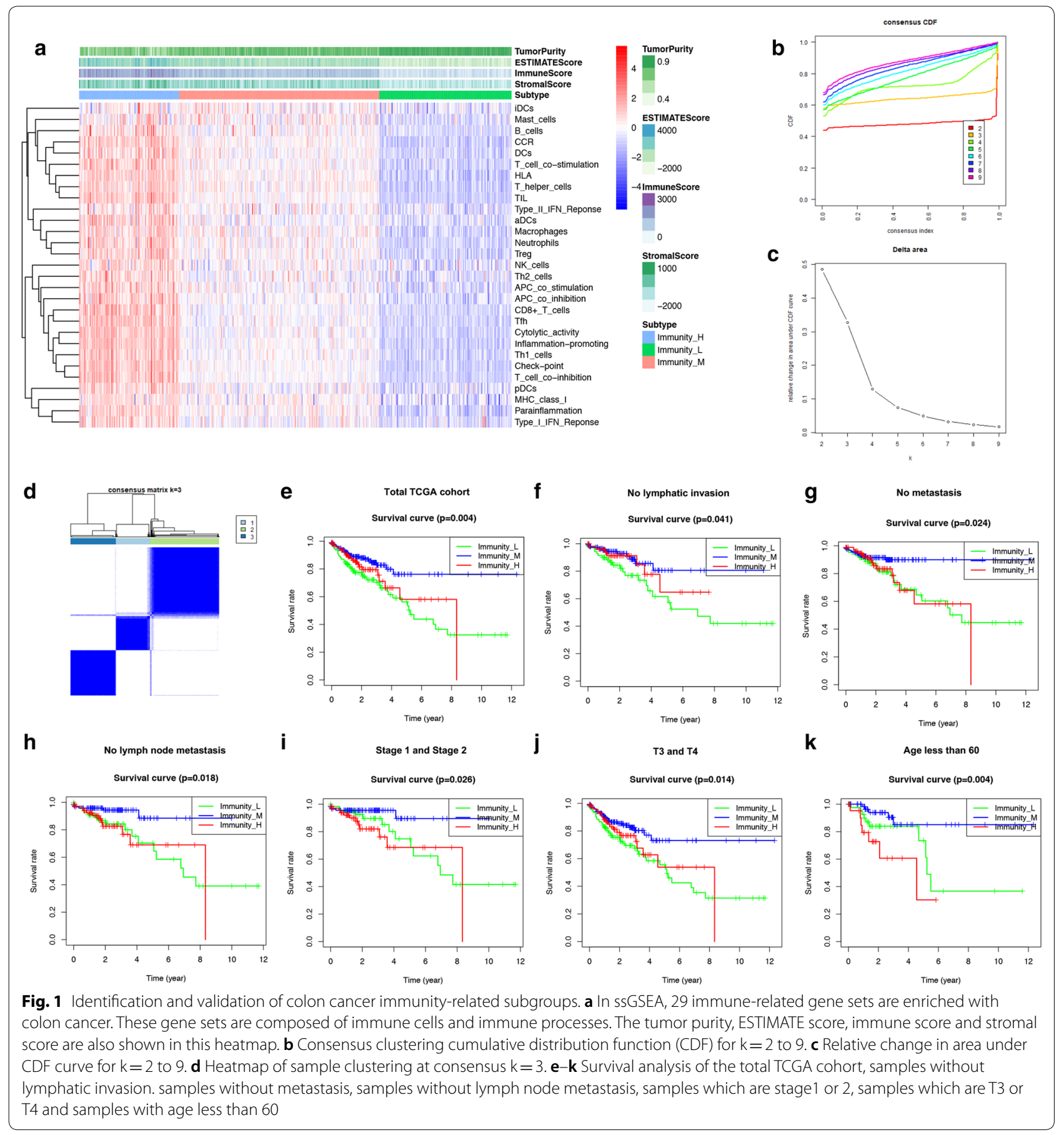

immunity group has medium expression level of those 4 genes. As a result, patients in high immunity group may be more sensitive to immune checkpoint-inhibiting agents, such as PD1 inhibitors and CTLA-4 inhibitors. The different landscape of immune cell infiltrating could also give ideas for immunotherapy, as high immunity group has higher level of T cells (CD4 T cell, CD8 T cell and $\mathrm{T}$ cell follicular helper) infiltration while low immunity group has higher level of macrophages $\mathrm{M} 0$, mast cell and NK cell infiltration. 


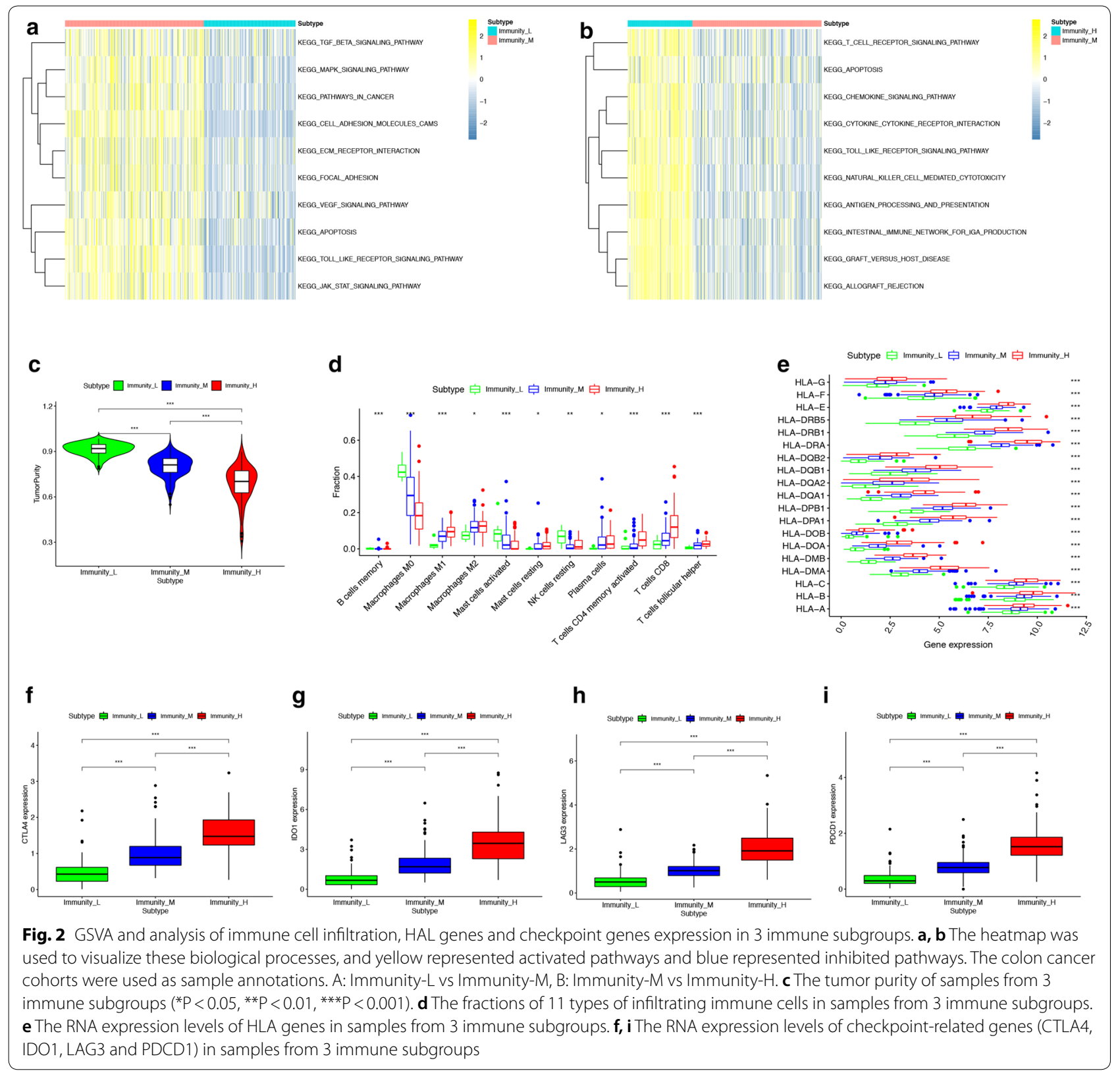

\section{Detection of immunity-related module and 8 hub genes by WGANA}

In WGCNA analysis, we identified 8 co-expression modules and analyzed their association with 10 clinical phenotypes, including fustat, TNM classification, stage, age, gender, lymphatic invasion, venous invasion and immunity (high, median and low) (Fig. 3a, b, Additional file 3: Figure S3). Except the grey module which contained nonclustering genes, the brown module was the most correlated module of immunity $(r=0.18, P=1 e-04$, Fig. $3 c)$. There were 212 genes in the brown module (Additional file 4: Table S1). The brown module was also correlated with $\mathrm{T}(\mathrm{r}=0.099, \mathrm{P}=0.04), \mathrm{N} \quad(\mathrm{r}=0.13, \mathrm{P}=0.007)$, stage $(r=0.099, P=0.04)$ and venous invasion $(r=0.11$, $\mathrm{P}=0.03)$. In the module-trait analysis, 8 genes with GS value $>0.3$ and $M M$ value $>0.8$ were defined as hub genes: CCDC69, CLMP, FAM110B, FAM129A, GUCY1B3, PALLD, PLEKHO1 and STY11. The GS values and MM values of 8 hub genes were shown in Additional file 5: Table S2. These hub genes were selected for further analysis. We validated the correlation between the relative infiltrating level of immune cells and the expression level of hub genes by the TIMER database (Additional file 6: Figure S4). The result demonstrated that the expression of 


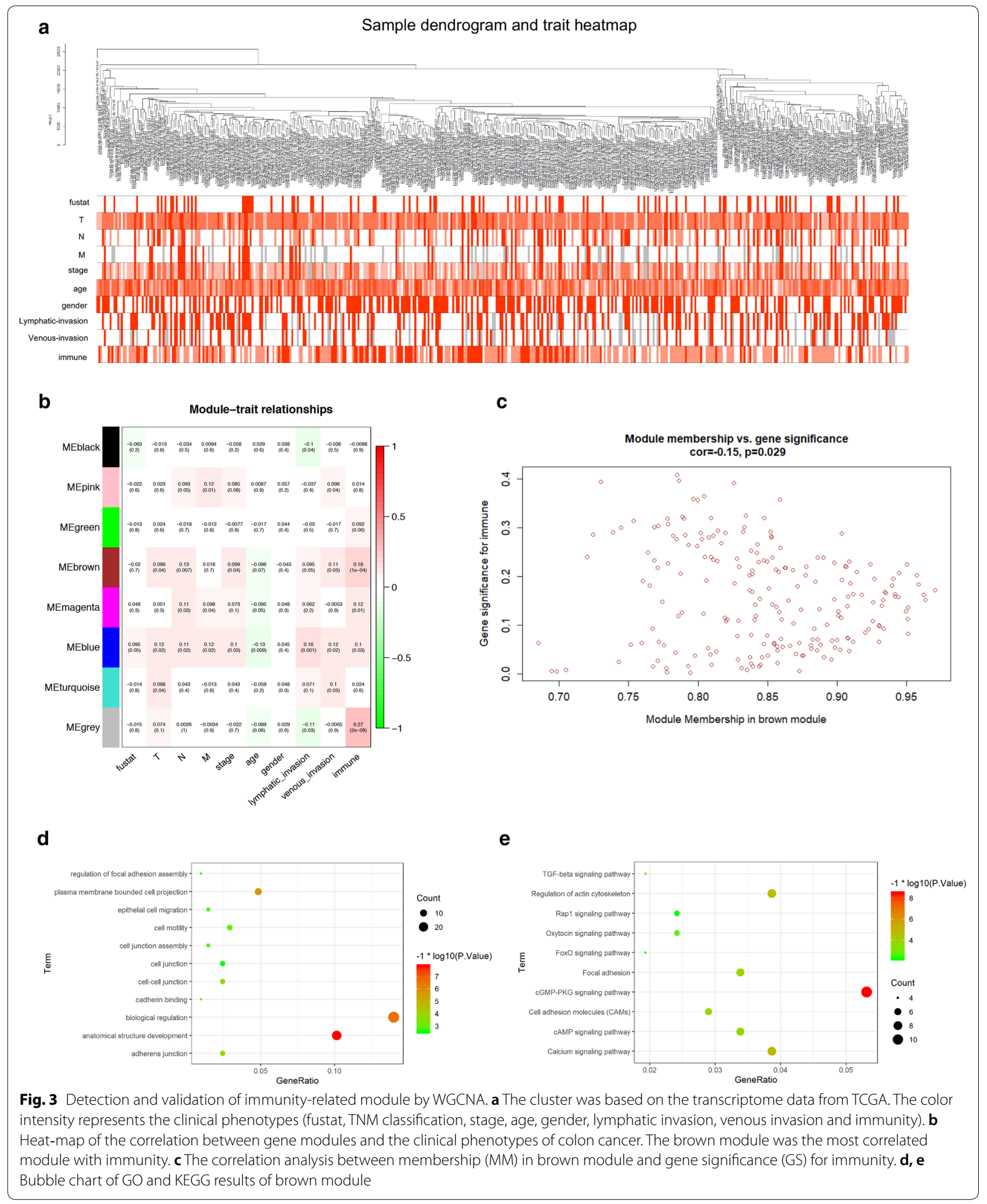


8 hub genes have negative correlation with tumor purity and their expression level were positively correlated with the infiltration of CD4 T cells, macrophages, neutrophils and dendritic cells. To investigate the underlying mechanism of the immunity-related module, we performed GO and KEGG analysis (Fig. 3d, e). In GO analysis, GO terms such as biological regulation, anatomical structure development and plasma membrane bounded cell projection were enriched in the brown module. In KEGG pathway analysis, cGMP-PKG, calcium and cAMP signaling pathways are also enriched with the brown module.

\section{Prognosis value and underlying mechanism of hub genes} Using transcriptome data from TCGA, we noticed that 7 of 8 hub genes are differentially expressed in colon cancer tissue and normal solid tissue (Fig. $4 a-h)$. And all of them have lower expression level in cancer tissues. We validated the protein expression of these hub genes based on IHC samples provided by the Human protein Atlas database. Compared to normal tissues, 6 of 8 hub genes were over-expressed in tumor tissues
(Fig. 5a-f). Clinical information analysis indicated that PALLD was correlated with venous invasion $(\mathrm{P}=0.047$, Fig. 4i), PLEKHO1 was correlated with lymphatic invasion $(P=0.019$, Fig. 4j) and SYT11 was correlated with lymph node metastasis $(\mathrm{P}=0.048$, Fig. $4 \mathrm{k})$. We annotated 8 hub-genes in Fig. 6a and used Metascape database to explore the function of these hub-genes (Fig. 6b, c). These genes were related to negative regulation of leukocyte activation and immune effector process, positive regulation of JAK-STAT signaling pathway, leukocyte apoptosis and granulocyte migration. Then we performed GSEA to explore the underlying mechanism of hub genes by assessing the enrichment of cancer hallmark gene sets (Additional file 7: Figure S5). The high-expression of most hub genes were enriched with epithelial mesenchymal transition, IL2-STAT signaling, IL6-JAK-STAT3 signaling, inflammatory response and KRAS signaling. Interestingly, these hallmarks are recognized to be related with immune reaction, progression of cancer and immunotherapy in some extent. In addition, the down-expression of these genes were
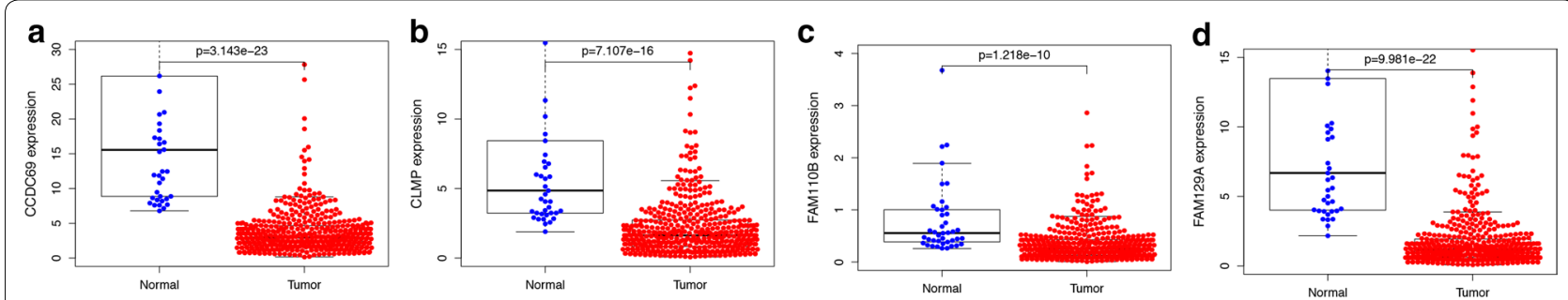

e

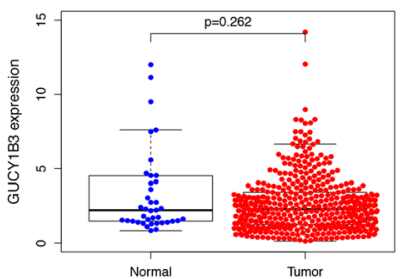

f

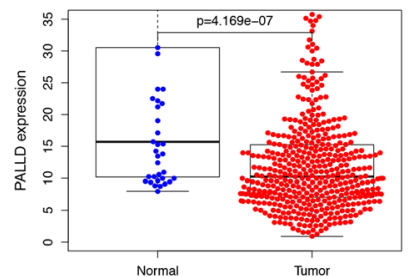

g

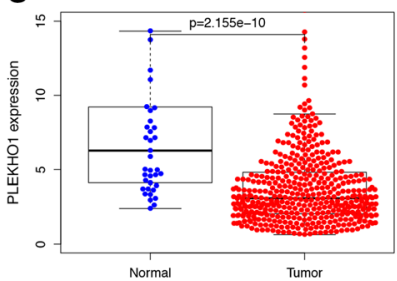

h

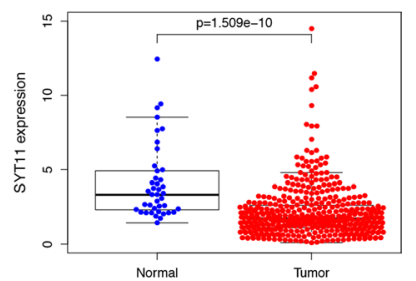

i

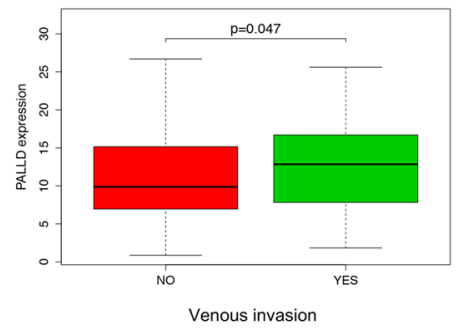

j

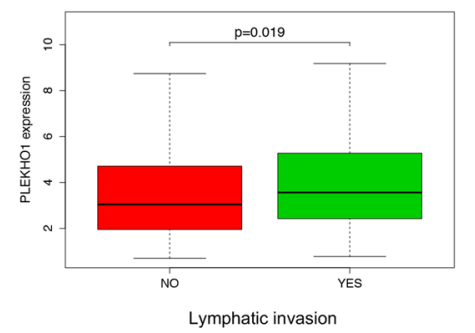

$\mathbf{k}$

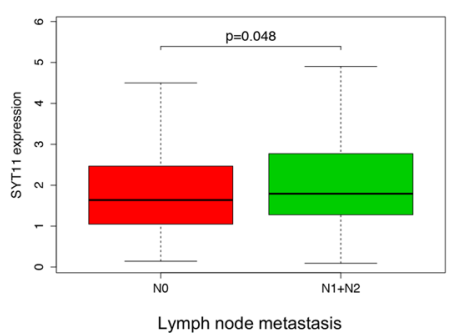

Fig. 4 Mann-Whitney test of 8 hub genes expression in different types of samples. $\mathbf{a}-\mathbf{h}$ Expression level of 8 hub genes in normal tissue and tumor tissue. $\mathbf{i}, \mathbf{j}$ The expression levels of hub genes are different in clinical subgroups. PALLD was correlated with venous invasion (P $=0.047)$, PLEKHO1 was correlated with lymphatic invasion $(P=0.019)$ and SYT11 was correlated with lymph node metastasis $(P=0.048)$ 
a

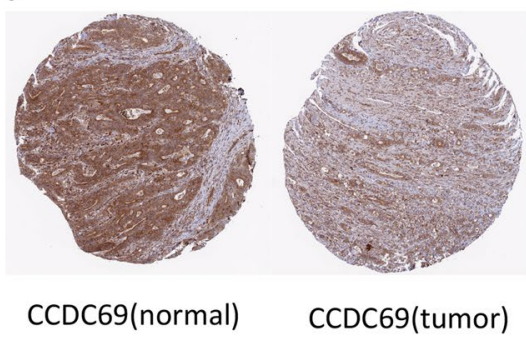

b

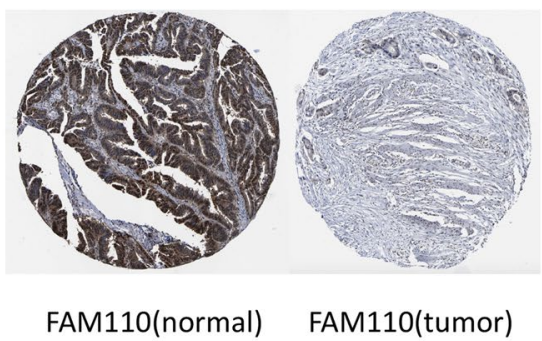

C

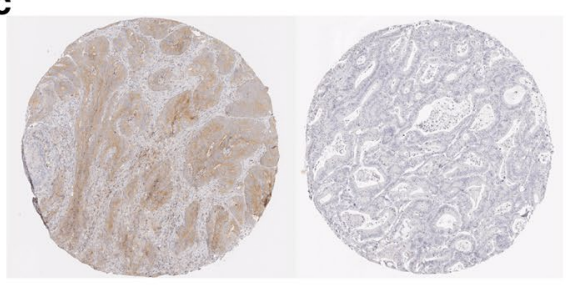

FAM129A(normal) FAM128A(tumor) d
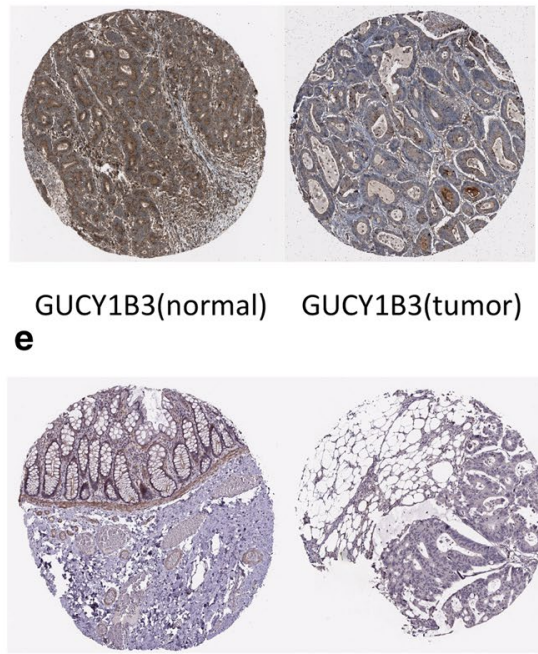

PALLD(normal) PALLD(tumor)

f

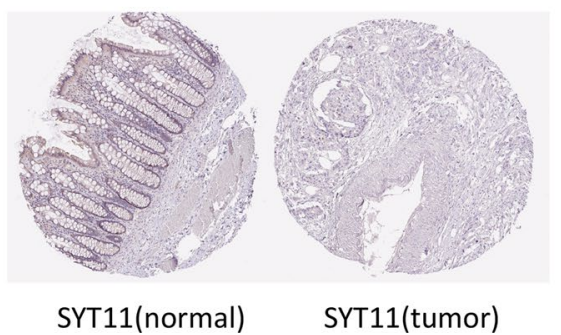

Fig. 5 The expressional differences of hub gene levels between colon cancer tissues and the para-cancer normal solid tissues in the Human Protein Atlas database

enriched with MYC target, which was also an important oncogene target in cancer development.

\section{TMB landscape in colon cancer and its correlation with immunity}

The TMB of samples from 3 immune subgroups were compared by Mann-Whitney test, indicating that tumors with higher immunity have higher TMB (Fig. 7a). The MSI status (proportion of MSI-H and MSI-L/MSS) were compared among 3 immune subgroups by Chi-square test (Fig. 7b). The exclusive and coincident associations across mutated genes were shown in Fig. 7c. These mutations were further classified into different categories: missense mutation, delectation, nonsense mutation, splice site, insertion, translation start site and nonstop mutation (Fig. 7d). For variant types, single nucleotide polymorphism (SNP) had a higher frequency than insertion or deletion (Fig. 7e), and $\mathrm{C}>\mathrm{T}$ was the most common single nucleotide variants (SNV) (Fig. 7f). Besides, we counted the number of altered bases in each sample and showed mutation types in box plot (Fig. $7 \mathrm{~g}-\mathrm{h}$ ). Finally, we exhibited the top 10 mutated genes in colon cancer with ranked percentages, including TTN (47\%), APC (75\%), MUC16 (27\%), SYNE1 (29\%), TP53 (55\%), KRAS (43\%), FAT4 (23\%), RYR2 (21\%), PIK3CA (28\%) and ZFHX4 (21\%) (Fig. 7i). Muation information of each sample in 3 immune subgroups (low immunity: $\mathrm{n}=119$, median immunity: $\mathrm{n}=179$, high immunity: $\mathrm{n}=88$ ) was exhibited in waterfall plot (Fig. 8a-c). We founded that the proportion of samples with specific mutated genes was different among 3 immune subtypes, which may provide suggestion for clinical application of immunotherapy.

\section{Discussion}

For colon cancer, patients with same clinical phenotypes can have different prognosis. As the relationship between chronic inflammation and colon cancer had been well demonstrated, more and more people began to study the role of immunity in cancer progression and considered it as one possible prognostic factor. In this study, we depicted the immune landscape of colon cancer using transcriptome and clinical information downloaded 


\begin{tabular}{|c|c|}
\multicolumn{2}{|l|}{$\mathbf{a}$} \\
\hline Gene Names & Entry \\
\hline CCDC69 & A6NI79 \\
CLMP & Q9H6B4 \\
\hline FAM110B & Q8TC76 \\
\hline FAM129A & Q9BZQ8 \\
\hline GUCY1B3 & Q02153 \\
PALLD & Q8WX93 \\
\hline PLEKHO1 & Q53GL0 \\
STY11 & Q8RWL6 \\
\hline
\end{tabular}

\section{b}
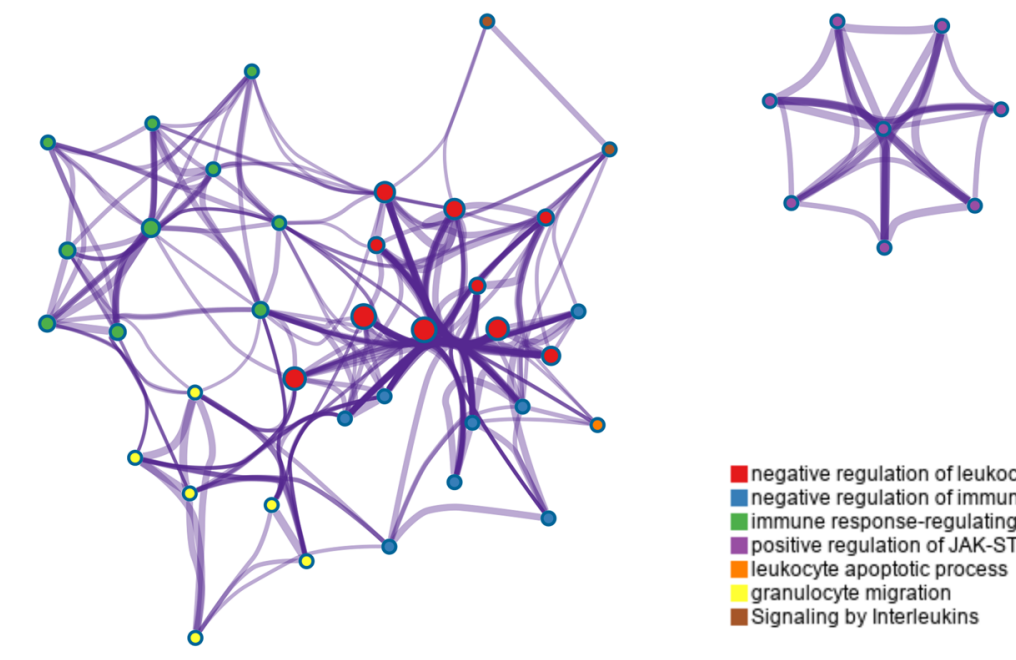

Protein Niban 1 (Cell growth-inhibiting gene 39 protein)

Guanylate cyclase soluble subunit beta- 1

$$
\text { Palladin }
$$

Pleckstrin homology domain-containing family $\mathrm{O}$ member 1

serine/threonine-protein kinase 11

\begin{tabular}{|l} 
Length \\
\hline 296 \\
373 \\
370 \\
928 \\
619 \\
1383 \\
409 \\
570
\end{tabular}

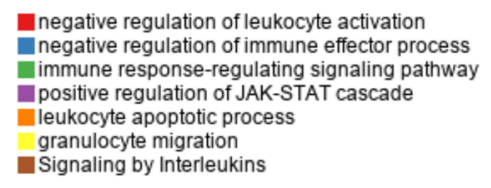

C

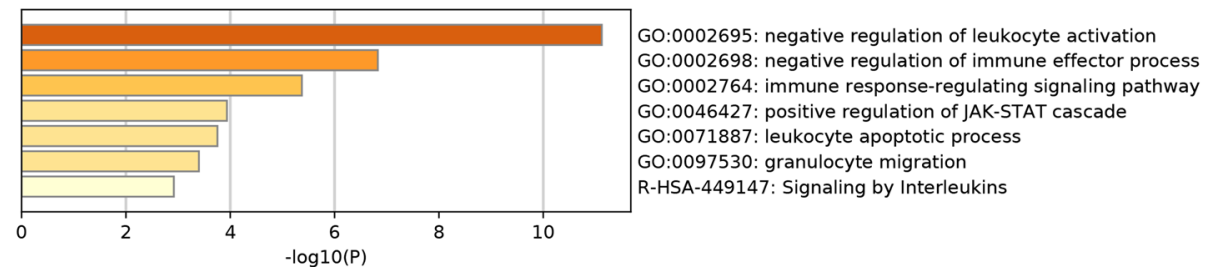

Fig. 6 Functional analysis of hub genes. a The annotation of hub genes using Uniport database. b Protein-Protein interaction network of genes which were directly related to hub genes. A sport represented a gene and the color of spots represented which pathway this gene was involved in. C The enrichment statistical significance of GO-terms and KEGG pathways

from the TCGA database. The colon cancer samples were clustered into three clusters (low immunity, median immunity and high immunity). The patients in the median-immunity cluster had the best prognosis compared with patients in the low-immunity and high-immunity clusters. Patrick Danaher et al. established a tumor inflammation signature based on 30 types of cancers and found that high immunity was related to better prognosis in skin cutaneous melanoma and sarcoma, while low immunity was related to better prognosis in pancreatic adenocarcinoma and lower grade glioma. These findings were different from ours, which demonstrated heterogeneity in immune landscape among different cancers. Generally, cytotoxic T cell (CD8+T cell) immune response is considered to have anti-tumor effects by IFN$\gamma$, TNF- $\alpha$ and IL17. As a result, increased T cell infiltration in tumor tissue may lead to an anti-tumor effect in the high-immunity group. But in our study, the patients in the median immunity cluster had the best prognosis compared with patients in the low immunity and high immunity clusters. Robert D. et al. concluded that the immunity mainly plays three roles in anti-tumor effects: prevents the establishment of inflammatory, protects the host from viral infection, kills tumor cells in specific tissue. However, cytokines such as IL-12 and interferon- $\gamma$ (IFN- $\gamma$ ) can contribute to the construction of immunoediting and immune escape [35]. Convincingly, immunity can also provide the selective pressure 

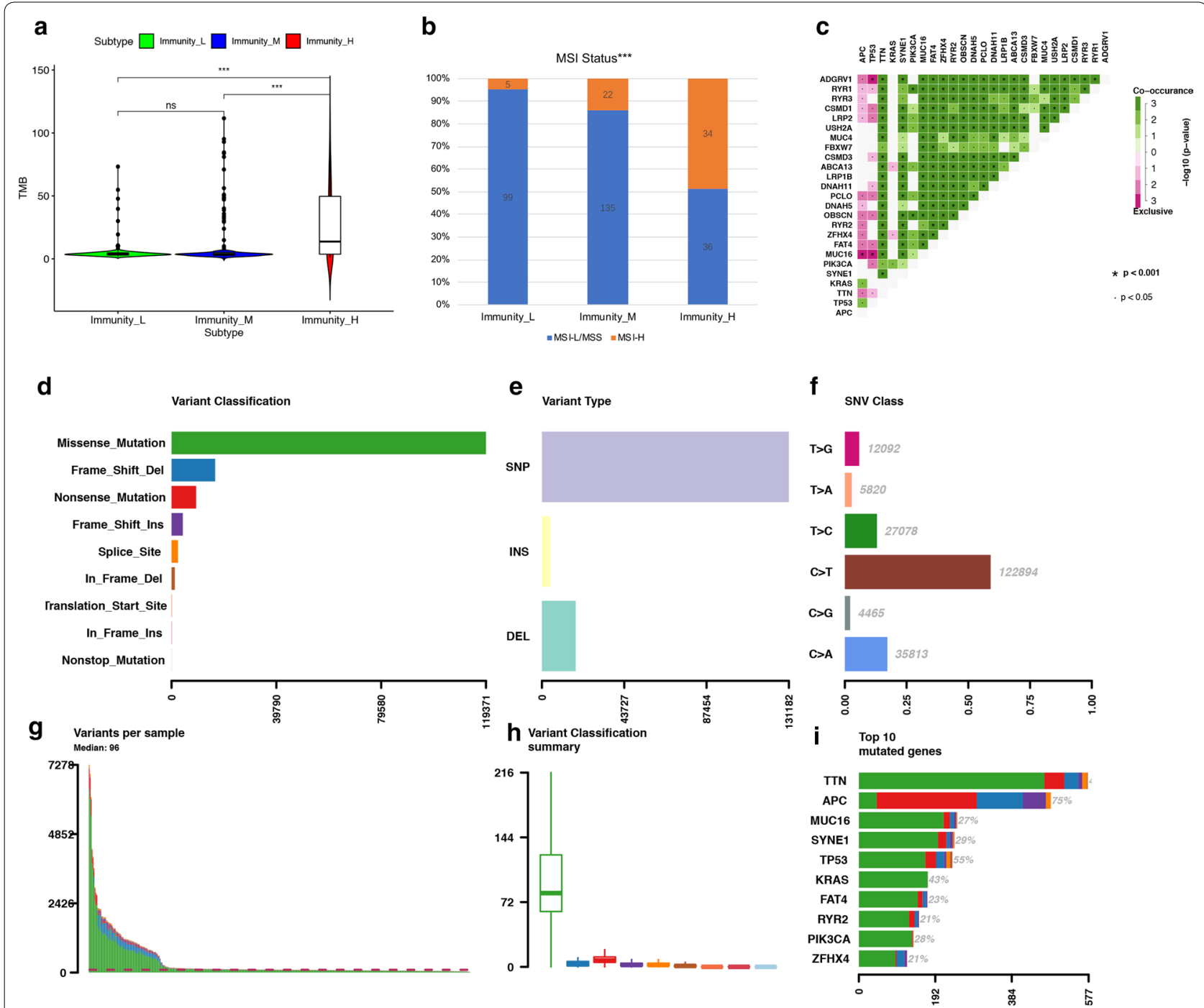

Fig. 7 The landscape of frequently mutated genes in colon cancer. a The TMB of samples from 3 immune subgroups $\left({ }^{*} P<0.05\right.$, ${ }^{* *} P<0.01$, $\left.{ }^{* * *}>0.001\right)$. b The Chi-square test of MSI status in 3 immune subgroups. $\mathbf{c}$ The coincident and exclusive associations across mutated genes. $\mathbf{d}$ Classification and frequency of mutation types. e Frequency of variant types. $\mathbf{f}$ Frequency of SNV classes. $\mathbf{g}, \mathbf{h}$ tumor mutation burden in specific samples; $\mathbf{i}$ the top 10 mutated genes in colon cancer

that accelerates the proliferation of tumor cells which have gained immune-evasive mutations [36]. Our TMB analysis showed that the high immunity cluster had the highest mutational burden which also provide evidence for the establishment of immune-evasive mutations and further immune escape. So, it is arbitrary to conclude that patient with higher immunity can have better prognosis. This finding could explain that patients who keep an equilibrium between immune elimination and immunoediting may have better prognosis.

By using WGCNA, we obtained 8 hub genes which occupied important positions in the immune mechanism of colon cancer. These hub genes had lower transcriptional expression levels in tumor tissue than normal tissue. In addition, by analyzing transcriptome data from TCGA, the RNA translational levels of seven hub genes have statistical difference in tumor and normal tissues. FAM110B has been proved to have an essential role in multiple cancer hallmarks and progression of many types of cancer such as prostate cancer [37]. FAM129 can affect invasion and proliferation by regulating autophagy, unfolded protein response and FAK signaling pathway [38]. The differential expression of GUCY1B3 has also been detected in breast cancer and ovarian cancer and though to inhibit tumor angiogenesis [39]. Ma et al. reported that long noncoding RNA 


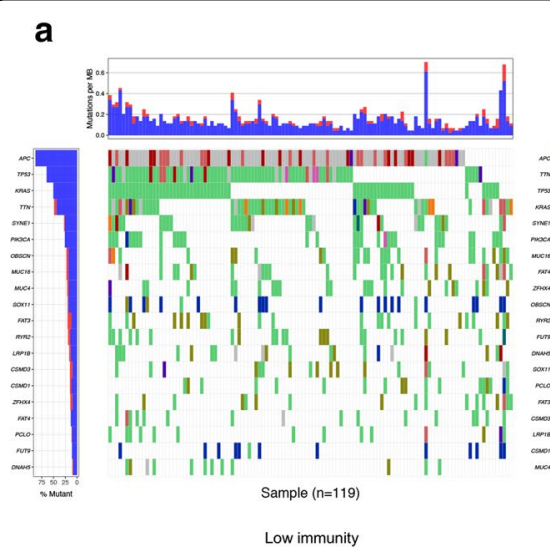

b

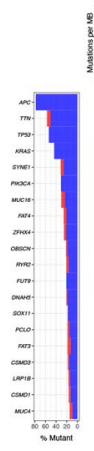

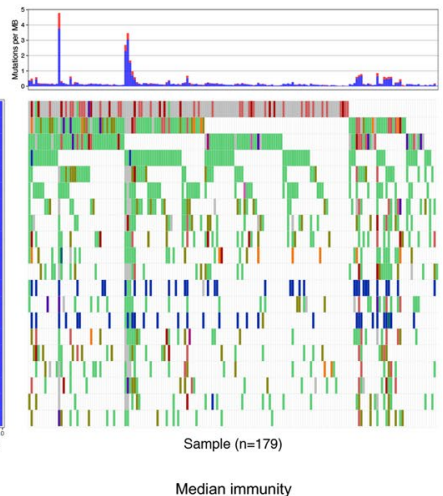

c

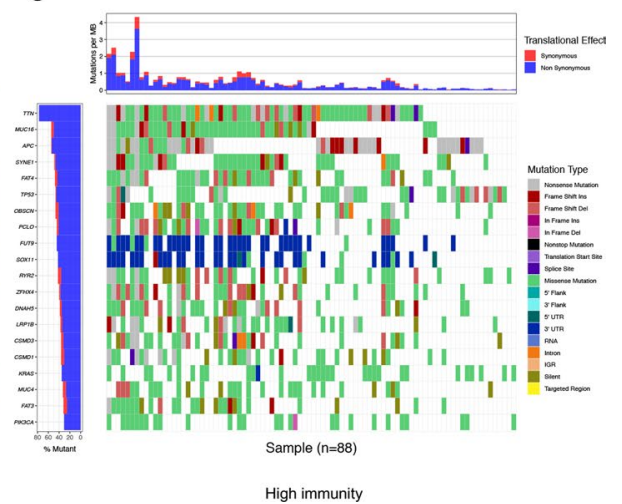

Fig. 8 Frequently mutated genes in 3 immune subgroups. a-c Waterfall plots display the frequently mutated genes in 3 immune subgroups of colon cancer. The left panel shows the genes ordered by their mutation frequencies. The right panel presents different mutation types

DUXAP8 can promote tumor progression by silencing PLEKHO1, revealing the anti-tumor effect of PLEKHO1 expression [40]. In GO and KEGG analysis, we noticed that immunity-related module had strong correlation with anatomical structure development and plasma membrane bounded cell projection. CLMP has been known as a new component of epithelial tight junctions, which support the function of hub genes in tumor cell metastasis [41]. And the result from Metascape database also proved that 8 hub-genes can have negative regulation on immune system. In further study, we need to perform deeper exploration in mechanisms and biofunctions of these hub genes, as their relationships with colon cancer have been seldomly reported.

The result of GSEA and TMB analysis indicated that high immunity was correlated with KRAS signaling pathway and high frequency of KRAS gene mutation. The expression levels of HLA genes and checkpoint genes (PD1/PDL1, CTLA4, IDO1 and LAG3) are higher in high immunity subgroup than median and low immunity subgroups, which convinced that our classification strategy were capable to provide support for immunotherapy. It has been reported that KRAS mutations could predict the resistance to epidermal growth factor receptor (EGFR) inhibitors such as cetuximab [42]. The prognostic value of KRAS mutations may be influenced by many factors, including primary tumor site, tumor stage, and adjuvant treatment received [21, 43]. Besides, stage 2 colon cancer patients with KRAS mutation were also reported to have increased risk of recurrence which was not affected by adjuvant chemotherapy. In the TMB analysis, classical tumor-related genes APC, TTN and TP53 also showed high mutational frequency among 3 immune subgroups. It is convinced that either polyposis or nonpolyposis syndromes can contribute to the genetic vulnerability to colon cancer, which is associated with mutation or loss of APC gene and several DNA mismatch repair genes [44, 45]. Xingyu Cheng et al. suggested that TTN and TP53 double mutation may participate in tumorigenesis by regulating downstream pathways with the participation of other co-expressed genes on the signaling network [46]]. In the future, it is of great significance to apply highly mutated genes and their correlated signaling pathways to searching for new targets for immunotherapy.

\section{Conclusion}

In this study, we divided patients from the TCGA cohort into three immune subgroups (high, median and low immunity) by applying unsupervised consensus clustering analysis. Three groups were different in survival outcome, stage, metastasis, lymph node metastasis, immune cell infiltration and expression levels of HLA and checkpoint genes. Then we performed WGCNA and got 8 hub genes (CCDC69, CLMP, FAM110B, FAM129A, GUCY1B3, PALLD, PLEKHO1 and STY11), which were in the module correlated with immune capacity. In functional analysis, we found that immunity was related to signaling pathways, such as inflammatory response and KRAS signaling pathway. Finally, we indicated that immunity was positively correlated with TMB and the mutational frequency of genes were significantly different among 3 immune subgroups.

\section{Supplementary information}

Supplementary information accompanies this paper at https://doi.
org/10.1186/s12967-020-02491-W.

Additional file 1: Figure S1. Survival analysis of colon cancer samples in different clinical subgroups. (A-H) Comparation of overall survival rate of 3 immune subgroups in different clinical subgroups (venous invasion, female, male, lymphatic invasion, M1, N1+N2, stage3+stage4, T1+T2). 
In all of these subgroups, there were no statistical differences among 3 immune subgroups in survival rate.

Additional file 2: Figure S2. Correlation between immunity and clinical phenotypes. The Chi-square test was performed to analyze the correlation between immunity (low, median and high) and clinical phenotypes (fustat, TNM classification, stage, age, gender, lymphatic invasion, venous invasion and immunity). We found that immunity was correlated with fustat, stage, $\mathrm{M}$ and $\mathrm{N}\left({ }^{*} \mathrm{P}<0.05,{ }^{* *} \mathrm{P}<0.01,{ }^{* * *} \mathrm{P}<0.001\right)$.

Additional file 3: Figure S3. WGCNA analysis of colon cancer based on TCGA transcriptome data. (A) Hierarchical cluster analysis was performed to detect co-expression modules with corresponding colors. (B-C) Softthresholding power analysis was used to obtain the scale-free fit index of network topology.

Additional file 4: Table S1 The list of genes in the brown module. Additional file 5: Table S2 The GS and MM values of hub genes.

Additional file 6: Figure S4 Validating the correlation between hub genes and immune cell infiltration. (A-H) We use the TIMER database to validate the correlation between the expression level of hub genes and the infiltration level of B cells, CD8+ cells, CD4+ cells, macrophages, neutrophils and dendritic cells in colon cancer tissues. The coefficient values and $P$ values were calculated by Spearman coefficient.

Additional file 7: Figure S5 GSEA of hub genes. (A-H) The high-expression of most hub genes were enriched with epithelial mesenchymal transition, IL2-STAT signaling, IL6-JAK-STAT3 signaling, inflammatory response and KRAS signaling. The down-expression of these genes were enriched with MYC target $\mathrm{V} 1$ and $\mathrm{V} 2$.

\section{Abbreviations}

TIM: Tumor immune microenvironment; TMB: Tumor mutational burden; ssGSEA: Single-Sample Gene Set Enrichment Analysis; WGCNA: Weighted correlation network analysis; TIM: Tumor immune microenvironment; PD1: Programmed death-1 receptor; CTLA-4: Cytotoxic T-lymphocyte antigen 4; LAG3: Lymphocyte activation gene-3; IDO1: Indoleamine 2, 3-dioxygenase 1; MAF: Mutation Annotation Format; HLA: Human leukocyte antigens; TOM: Topological Overlap Matrix; MEs: Module eigengenes; GS: Gene significance; KEGG: Kyoto Encyclopedia of Genes and Gene Ontology; GO: Gene Ontology; GSEA: Gene set enrichment analysis; MSigDB: Molecular Signatures Database; FDR: False discovery rate; SNP: Single nucleotide polymorphism; SNV: Single nucleotide variants; IFN- $\gamma$ : Interferon- $\gamma$.

\section{Acknowledgements}

The authors thank all the researchers and staff who supported the The Cancer Genome Atlas Research Network.

\section{Authors' contributions}

QGJ, XYW and JZDM designed the study. XYW and JZDM collected the mRNA transcriptome data and clinical information from TCGA. XRF and XYW performed analyses on TCGA data. QGJ and TYL performed statistical analyses. QGJ wrote the manuscript. QGJ, XYW, JZDM, XRF and TYL reviewed and revised the manuscript. All authors read and approved the final manuscript.

\section{Funding}

This work was supported by a grant from National Natural Science Foundation of China (Nos: 81560397 and 81660403).

\section{Availability of data and materials}

All analyzed data are accessible online, and the results of this article are included within the article as well as in additional files.

\section{Ethics approval and consent to participate}

Not applicable.

\section{Consent for publication}

Not applicable.

\section{Competing interests}

The authors declare that they have no competing interests.

\section{Author details}

${ }^{1}$ Department of Gastrointestinal Surgery, The First Affiliated Hospital of Nanchang University, Nanchang, Jiangxi, People's Republic of China. ${ }^{2}$ Queen Mary college, Medical Department, Nanchang University, Nanchang, Jiangxi, China.

Received: 4 March 2020 Accepted: 20 Auqust 2020

Published online: 28 August 2020

\section{References}

1. Chen W, Zheng R, Zhang S, Zeng H, Xia C, Zuo T, et al. Cancer incidence and mortality in China, 2013. Cancer Lett. 2017;401:63-71.

2. Labianca R, Beretta GD, Kildani B, Milesi L, Merlin F, Mosconi S, et al. Colon cancer. Crit Rev Oncol Hematol. 2010;74(2):106-33.

3. Pagès F, Mlecnik B, Marliot F, Bindea G, Ou F-S, Bifulco C, et al. International validation of the consensus Immunoscore for the classification of colon cancer: a prognostic and accuracy study. Lancet. 2018;391(10135):2128-39.

4. Beane J, Spira A, Lenburg ME. Clinical impact of high-throughput gene expression studies in lung cancer. J Thor Oncol. 2009;4(1):109-18.

5. Nagy Á, Lánczky A, Menyhárt O, Győrffy B. Validation of miRNA prognostic power in hepatocellular carcinoma using expression data of independent datasets. Sci Rep. 2018;8(1):9227.

6. Binnewies M, Roberts EW, Kersten K, Chan V, Fearon DF, Merad M, et al. Understanding the tumor immune microenvironment (TIME) for effective therapy. Nat Med. 2018;24(5):541-50.

7. Gajewski TF, Schreiber H, Fu Y-X. Innate and adaptive immune cells in the tumor microenvironment. Nat Immunol. 2013;14(10):1014-22.

8. Yaghoubi N, Soltani A, Ghazvini K, Hassanian SM, Hashemy SI. PD-1/ PD-L1 blockade as a novel treatment for colorectal cancer. Biomed Pharmacother. 2019;110:312-8.

9. Duraiswamy J, Kaluza KM, Freeman GJ, Coukos G. Dual blockade of PD-1 and CTLA-4 combined with tumor vaccine effectively restores T-cell rejection function in tumors. Cancer Res. 2013;73(12):3591-603.

10. Nguyen LT, Ohashi PS. Clinical blockade of PD1 and LAG3 - potential mechanisms of action. Nat Rev Immunol. 2015;15(1):45-56.

11. Zhai L, Ladomersky E, Lenzen A, Nguyen B, Patel R, Lauing KL, et al. IDO1 in cancer: a Gemini of immune checkpoints. Cell Mol Immunol. 2018;15(5):447-57.

12. Goodman AM, Kato S, Bazhenova L, Patel SP, Frampton GM, Miller $V$, et al. Tumor mutational burden as an independent predictor of response to immunotherapy in diverse cancers. Mol Cancer Ther. 2017;16(11):2598-608.

13. Miao D, Margolis CA, Vokes NI, Liu D, Taylor-Weiner A, Wankowicz SM, et al. Genomic correlates of response to immune checkpoint blockade in microsatellite-stable solid tumors. Nat Genet. 2018;50(9):1271-81.

14. Samstein RM, Lee $\mathrm{C}-\mathrm{H}$, Shoushtari AN, Hellmann MD, Shen R, Janjigian $Y Y$, et al. Tumor mutational load predicts survival after immunotherapy across multiple cancer types. Nat Genet. 2019;51(2):202-6.

15. Boland CR, Goel A. Microsatellite instability in colorectal cancer. Gastroenterology. 2010;138(6):2073-87.

16. Arrington AK, Heinrich EL, Lee W, Duldulao M, Patel S, Sanchez J, et al. Prognostic and predictive roles of KRAS mutation in colorectal cancer. Int J Mol Sci. 2012;13(10):12153-68.

17. Prahallad A, Sun C, Huang S, Di Nicolantonio F, Salazar R, Zecchin D, et al. Unresponsiveness of colon cancer to BRAF(V600E) inhibition through feedback activation of EGFR. Nature. 2012;483(7387):100-3.

18. Lin L, Liu A, Peng Z, Lin H-J, Li P-K, Li C, et al. STAT3 is necessary for proliferation and survival in colon cancer-initiating cells. Cancer Res. 2011;71(23):7226-377.

19. Mayakonda A, Lin D-C, Assenov Y, Plass C, Koeffler HP. Maftools: efficient and comprehensive analysis of somatic variants in cancer. Genome Res. 2018;28(11):1747-56. 
20. Liu Y, Sethi NS, Hinoue T, Schneider BG, Cherniack AD, Sanchez-Vega F, et al. Comparative molecular analysis of gastrointestinal adenocarcinomas. Cancer Cell. 2018;33(4):721-35.

21. Barbie DA, Tamayo P, Boehm JS, Kim SY, Moody SE, Dunn IF, et al. Systematic RNA interference reveals that oncogenic KRAS-driven cancers require TBK1. Nature. 2009;"462(7269):108-12. https://www.nature.com/articles/ nature08460

22. Subramanian A, Tamayo P, Mootha VK, Mukherjee S, Ebert BL, Gillette $M A$, et al. Gene set enrichment analysis: A knowledge-based approach for interpreting genome-wide expression profiles. Proc Natl Acad Sci. 2005;102(43):15545-50.

23. Rooney MS, Shukla SA, Wu CJ, Getz G, Hacohen N. Molecular and genetic properties of tumors associated with local immune cytolytic activity. Cell. 2015;160(1-2):48-61.

24. Massink MPG, Kooi IE, Martens JWM, Waisfisz Q, Meijers-Heijboer H. Genomic profiling of CHEK2*1100delC-mutated breast carcinomas. BMC Cancer. 2015;15(1):877.

25. Bedognetti D, Hendrickx W, Marincola FM, Miller LD. Prognostic and predictive immune gene signatures in breast cancer. Curr Opin Oncol. 2015:27(6):433-44.

26. Aran D, Lasry A, Zinger A, Biton M, Pikarsky E, Hellman A, et al. Widespread parainflammation in human cancer. Genome Biol. 2016;17(1):145.

27. Wong HS-C, Chang C-M, Liu X, Huang W-C, Chang W-C. Characterization of cytokinome landscape for clinical responses in human cancers. Oncoimmunology. 2016;5(11):1214789.

28. Charoentong P, Finotello F, Angelova M, Mayer C, Efremova M, Rieder D, et al. Pan-cancer Immunogenomic Analyses Reveal Genotype-Immunophenotype Relationships and Predictors of Response to Checkpoint Blockade. Cell Rep. 2017;18(1):248-62.

29. Liu Z, Li M, Jiang Z, Wang X. A comprehensive immunologic portrait of triple-negative breast cancer. Transl Oncol. 2018:11(2):311-29.

30. De Simone M, Arrigoni A, Rossetti G, Gruarin P, Ranzani V, Politano $C$, et al. Transcriptional landscape of human tissue lymphocytes unveils uniqueness of tumor-infiltrating T regulatory cells. Immunity. 2016:45(5):1135-47.

31. Yoshihara K, Shahmoradgoli M, Martínez E, Vegesna R, Kim H, TorresGarcia W, et al. Inferring tumour purity and stromal and immune cell admixture from expression data. Nat Commun. 2013;4(1):2612. https:// www.nature.com/articles/ncomms3612

32. Newman AM, Liu CL, Green MR, Gentles AJ, Feng W, Xu Y, et al. Robust enumeration of cell subsets from tissue expression profiles. Nat Methods. 2015;12(5):453-7. https://www.nature.com/articles/nmeth.3337

33. Langfelder P, Horvath S. WGCNA: an R package for weighted correlation network analysis. BMC Bioinform. 2008;9(1):559. https://doi. org/10.1186/1471-2105-9-559.

34. Zhou Y, Zhou B, Pache L, Chang M, Khodabakhshi AH, Tanaseichuk O, et al. Metascape provides a biologist-oriented resource for the analysis of systems-level datasets. Nat Commun. 2019;10(1):1523.
35. Dunn GP, Bruce AT, Ikeda H, Old L, Schreiber RD. Cancer immunoediting: from immunosurveillance to tumor escape. Nat Immunol. 2002;3(11):991-8

36. Koebel CM, Vermi W, Swann JB, Zerafa N, Rodig SJ, Old LJ, et al. Adaptive immunity maintains occult cancer in an equilibrium state. Nature. 2007:450(7171):903-7.

37. Vainio $\mathrm{P}$, Wolf $\mathrm{M}$, Edgren $\mathrm{H}, \mathrm{He}$, Kohonen $\mathrm{P}$, Mpindi J-P, et al. Integrative genomic, transcriptomic, and RNAi analysis indicates a potential oncogenic role for FAM110B in castration-resistant prostate cancer. Prostate. 2012;72(7):789-802.

38. Nozima BH, Mendes TB, Pereira GJ, Araldi RP, Iwamura ESM, Smaili SS, et al. FAM129A regulates autophagy in thyroid carcinomas in an oncogenedependent manner. Endocr Relat Cancer. 2019;1:227-38.

39. Mohammadoo khorasani M, Karami Tehrani F, Parizadeh SMR, Atri M. Differential expression of alternative transcripts of soluble guanylyl cyclase, GYCY1a3 and GUCY1b3 genes, in the malignant and benign breast tumors. Nitric Oxide. 2019;83(4):65-71.

40. Ma H-W, Xie M, Sun M, Chen T-Y, Jin R-R, Ma T-S, et al. The pseudogene derived long noncoding RNA DUXAP8 promotes gastric cancer cell proliferation and migration via epigenetically silencing PLEKHO1 expression. Oncotarget. 2017;8(32):338-42.

41. Raschperger E, Engstrom U, Pettersson RF, Fuxe J. CLMP, a novel member of the CTX family and a new component of epithelial tight junctions. J Biol Chem. 2004;279(1):796-804.

42. Huang L, Fu L. Mechanisms of resistance to EGFR tyrosine kinase inhibitors. Acta Pharm Sin B. 2015;5(5):390-401.

43. Muzny DM, Bainbridge MN, Chang K, Dinh HH, Drummond JA, Fowler $\mathrm{G}$, et al. Comprehensive molecular characterization of human colon and rectal cancer. Nature. 2012;487(7407):330-7.

44. Lazarus J, Maj T, Smith JJ, Perusina Lanfranca M, Rao A, D'Angelica MI, et al. Spatial and phenotypic immune profiling of metastatic colon cancer. JCl Insight. 2018:3:22.

45. Kwong LN, Dove WF. APC and its modifiers in colon cancer. In: Advances in experimental medicine and biology. 2009. p. 85-106.

46. Cheng X, Yin H, Fu J, Chen C, An J, Guan J, et al. Aggregate analysis based on TCGA:TTN missense mutation correlates with favorable prognosis in lung squamous cell carcinoma. J Cancer Res Clin Oncol. 2019:145(4):1027-35.

\section{Publisher's Note}

Springer Nature remains neutral with regard to jurisdictional claims in published maps and institutional affiliations.
Ready to submit your research? Choose BMC and benefit from:

- fast, convenient online submission

- thorough peer review by experienced researchers in your field

- rapid publication on acceptance

- support for research data, including large and complex data types

- gold Open Access which fosters wider collaboration and increased citations

- maximum visibility for your research: over $100 \mathrm{M}$ website views per year

At BMC, research is always in progress.

Learn more biomedcentral.com/submissions 